This is an open-access article distributed under the terms of the Creative Commons Attribution License, which permits unrestricted use, distribution, and reproduction in any medium, provided the original author(s) and source are credited.

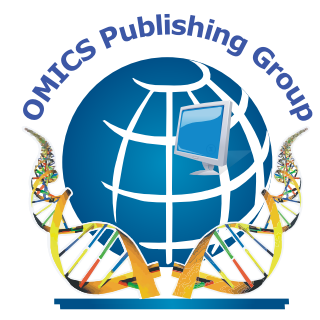

ISSN: 2165-7556

\title{
Journal of Ergonomics
}

\section{The International Open Access \\ Journal of Ergonomics}

\section{Editor-in-Chief}

Patrick E. Patterson

Texas University, USA

\section{Executive Editors}

\section{Masahiro Tomita}

Mie University, Japan

\section{Larry Fennigkoh}

Milwaukee School of Engineering, USA

Paul Atchley

University of Kansas, USA

Nancy A Nickman

University of Utah, USA

\section{Available online at: OMICS Publishing Group (www.omicsonline.org)}

7 his article was originally published in a journal by OMICS

1 Publishing Group, and the attached copy is provided by OMICS Publishing Group for the author's benefit and for the benefit of the author's institution, for commercial/research/educational use including without limitation use in instruction at your institution, sending it to specific colleagues that you know, and providing a copy to your institution's administrator.

All other uses, reproduction and distribution, including without limitation commercial reprints, selling or licensing copies or access, or posting on open internet sites, your personal or institution's website or repository, are requested to cite properly. 


\title{
Assessing the Effect of Self-positioning on Cognitive Executive Function
}

\author{
Schraefel MC ${ }^{1}$, Kenneth Jay ${ }^{1,2 *}$ and Lars L Andersen ${ }^{2}$
}

${ }^{1}$ Electronics and Computer Science, University of Southampton, United Kingdom

${ }^{2}$ National Research Centre for the Working Environment, Denmark

\begin{abstract}
Objective: The aim of this pilot study was to explore the effects of self-positioning on cognitive performance in the work environment using a standardized cognitive test battery to evaluate executive function under two conditions.

Methods: This randomized controlled cross-over trial involved 17 men (mean age \pm SD: $29.8 \pm 5.5$ ) all with a science background. The participants were accustomed to working in an open environment and none of whom currently using standing desks. We used a modified version of the CNS Vital Signs (CNSVS) test battery to assess cognitive executive function in two typical work positions - standing and seated. Participants were randomly assigned to a standing or seated position to begin the testing procedure. Upon completion of the first test round they were instructed to rest for $10 \mathrm{~min}$ in a dark room with no distractions before commencing the second round of testing positioned in the alternate fashion. The main outcome measure was a CNSVS score in each of the six cognitive executive function domains in the two different work positions.
\end{abstract}

Results: A two-tailed paired $t$-test showed a significant difference between the standing (mean+/-SD: $94 \pm 10$ ) and seated (mean \pm SD: $99 \pm 9$ ) position $(p<0.01)$ in the domain score of Complex Attention but not in the other cognitive executive function domains.

Conclusion: Self-positioning has a significant impact on cognitive executive function when Complex Attention is required. Considerations towards body positioning and task may be beneficial in the workplace to optimize cognitive performance and lower the risk of person-made mistakes.

Keywords: CNSVS; Design; Sitting; Standing; Complex attention; Knowledge workers; Executive function

\section{Introduction}

This past year an increasing number of articles in both research and the popular press have been suggesting that sitting at work all day causes a raft of harms from increasing likelihood of insulin [1] to shortening life expectancy [2]. The musculo-skeletal costs of sitting vs. standing have been investigated in research since the 70s looking at the effects of body posture on spinal joint loading and compression, joint kinematics of the spine and muscle trunk activation as primary focus areas [3-9]. For instance, Nachemson [7] reported 40 to 175 percent greater disc pressures in a variety of sitting postures, compared to standing. Standing appears to unload the passive tissue structures of the back thus providing a resting position from the seated posture [3]. More recent research has been translated into an increase of standing desks in the working environment, where standing is perceived to be an antidote to sitting when dealing with musculo- skeletal disorders [10]. In contrast, some researchers in human factors and ergonomics have also found adverse effects to standing [11] as well as to sit-stand conditions on musculo-skeletal disorders, alertness and performance [12].

When it comes to cognitive performance and executive function, the benefits of standing or sitting are less clear. Cognitive function largely involves the area of the brain known as the pre-frontal cortex, which is the anterior part of the frontal lobes of the brain, lying in front of the motor and premotor areas. The term Cognitive Executive Function is often used as an umbrella-term for cognitive activities such as planning, working memory, attention, problem solving, verbal reasoning, multi-tasking, and monitoring of actions among others and are processes localized in the pre-frontal cortex $[13,14]$.

Recent studies have evaluated the difference between sitting, and walking desks (where one walks on a treadmill fixed to a standing desk) [15], or sitting, standing and walking desks [16]. Intriguingly, while the trend seems to be that many attributes improve from standing [16] or at least do not degrade, the positives are not clearly universal. One study where various cognitive executive function tests have been used in these cases to gauge performance showed that certain tasks performed at walking desks, definitely performed more poorly [17]. Likewise the papers do not all use tests that study the same attributes of cognitive executive function. Some may focus on motor speed/motor control [16] rather than more involved cognitive executive function tasks that require Complex Attention or Cognitive Flexibility [17].

In the present study we look at broader range of cognitive executive function assessments than previous work to assess self-positioning (standing or sitting). Our goal is to gain a better picture of effects that could more directly inform human-computer interaction design of both digital information systems and their physical environments for knowledge work [18]. For instance, optimal self-positioning for motor control may be important for precision work in a laboratory, but may not be a key factor for managing email. Other cognitive executive function measures like attention and recall may be more important, or at least more descriptive for multi-tasking, a common element of most of our digital-physical lives. Therefore, the objective of this study is to investigate, in an exploratory fashion, cognitive performance using a

*Corresponding author: Kenneth Jay, National Research Centre for the Working Environment, Lers $\varnothing$ Parkalle 105, DK-2100 Copenhagen, Denmark, Tel: +4561701009; E-mail: kj@ecs.soton.ac.uk

Received August 24, 2012; Accepted November 27, 2012; Published November 30, 2012

Citation: Schraefel MC, Kenneth Jay, Andersen LL (2012) Assessing the Effect of Self-positioning on Cognitive Executive Function. J Ergonomics 2:110. doi:10.4172/2165-7556.1000110

Copyright: ( 2012 Schraefel MC, et al. This is an open-access article distributed under the terms of the Creative Commons Attribution License, which permits unrestricted use, distribution, and reproduction in any medium, provided the original author and source are credited. 
test battery of cognitive executive function tests under two conditions: standing and seated at a desk. Based on the physiological benefits of standing for people who are mainly seated knowledge workers, we hypothesize a general higher cognitive performance score across the six individual cognitive executive function domains in the standing position.

\section{Methods}

\section{Study design}

We conducted a randomized controlled cross-over trial to test the effect of position on cognitive task performance using standard computer display/interaction technology.

In short, the testing procedure had two almost identical parts with the only difference being the standing or seated position in randomized order.

The office for the study was set up with a standard height table and chair for the seated position, and boxes on the table for the standing condition (Figure 1). The same laptop, a Lenovo ThinkPad T41p running Windows XP, was used in each condition. For seated, the laptop was placed on the table; for standing, the laptop was placed on the rigid boxes at an appropriate ergonomic height for standing work. Participants were shown how to start the test battery on the laptop when they were ready to begin; the program guided them through each test, and indicated completion. Between test conditions, the laptop was repositioned for the next condition. The total test time including rest took an average of one hour and ten minutes.

\section{Participants}

We constrained the participant group to men (mean age \pm SD: 29.8 \pm 5.5 ), all healthy with science backgrounds and graduate degrees, and none of whom currently using standing desks; all participants $(n=17)$ worked in an open lab environment. Participants signed an information and consent form approved via the University of Southampton Ethical Review of Human Participant Studies process. (ID: 1497). Furthermore, participants received a gift voucher as part of their participation. We registered the study at clinicaltrials.gov (number NCT01641588).

\section{Cognitive executive function testing}

We used the CNS Vital Signs (CNSVS) test battery for assessing neurocognitive function. The included subtests were the following: Symbol Digit Coding test, Shifting Attention Test, Finger Tapping Test, Stroop Test, and Continuous Performance Test in that order. The tests themselves are online versions of standard measures of cognitive task performance and have previously been described and tested for reliability and validity in the literature. The test runs are highly consistently delivered: the test battery is automatically presented to the participants with practice followed by test runs of each test in the selected battery. In short, the subtests provide domain measures of: Executive Function, Complex Attention, Cognitive Flexibility, Psychomotor Speed, Reaction Time and Processing Speed. The score of each of the domains are calculated either as differences between correct and erroneous responses within each subtest, or summation of the responses from two or more subtests.

\section{Statistics}

We used a two-tailed paired Student's $t$-test to test for significant differences between the standing and seated condition. An alpha level of $<0.05$ was considered significant unless otherwise specified and we report results as means (SD). One participant was excluded from statistical analysis due to an invalid CNSVS test, thus bringing the total number of participants in the analysis to 16 .

\section{Results}

Table 1 shows the outcome scores of each of the six cognitive executive function domains tested in the two conditions. Higher domain scores indicate better cognitive function. Only the domain of Complex Attention was significantly different in the standing vs. seated condition (confidence interval: 1.66:9.96) with a Cohen's d-value of 0.79. A total of 13 of the 16 participants did better on Complex Attention tasks in the seated position.

\section{Discussion}

To our knowledge this is the first time the effects of self-positioning on cognitive performance have been tested with an extensive cognitive test battery consisting of several sub-tests to provide test scores in six different domains of cognitive executive function. Our exploratory study showed a significant better performance in testing the ability to keep a sustained focus, to resist distraction, to switch attention between tasks as well as information processing ability, attributes known as complex attention, when sitting down compared to standing.

For work environment design, the understanding of how such formal abstract tests of a single performance factor (sitting/standing) map to real-world interactions is required. For Complex Attention, for instance, where we found a significant difference between sitting and standing, a possible mapping between cognitive executive function assessment tasks and real life activity may be observed in the work of laboratory workers. For instance, Plebani and Carraro [19] found 189 laboratory mistakes out of 40.490 tests pooled from four different departments of a hospital. While a relative mistake frequency of $0.47 \%$ does not seem significant, $19 \%$ of the laboratory mistakes were associated with further inappropriate investigations. Similarly, $6.4 \%$ of the laboratory mistakes were associated with inappropriate care or modification of therapy, resulting in unjustifiable socioeconomic costs [19]. In a more recent study, Jequier and Ukombe [20] found a coefficient of variation of $44.3 \%$ when 26 medical laboratory technicians and pathologists performed a routine analysis on an aliquot of the same sample specimen, which they reported as being unacceptably high [20].

According to multiple surveys between 2001-2004 [21] and analyses of data from 2004-2008 [22], the knowledge economy involves anywhere from $30-57 \%$ of workers by occupation in the EU, and contributes at least $40 \%$ to GDP and growing. This growth makes

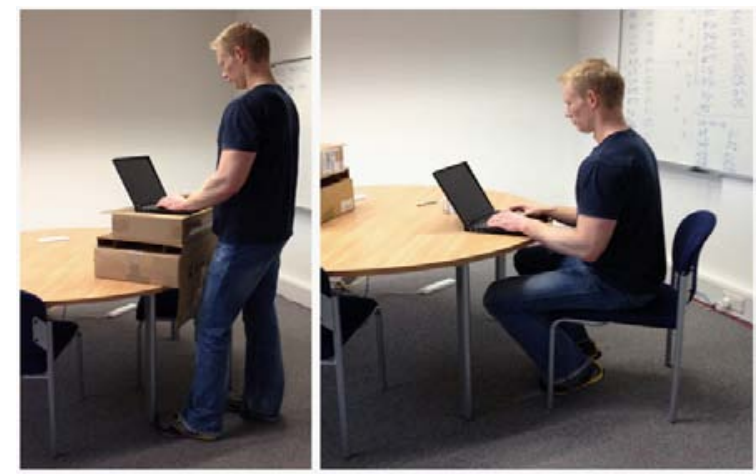

Figure 1: Test setup for the standing and seated conditions running the Cognitive Executive Function test battery on the study laptop. 


\begin{tabular}{|c|c|c|}
\hline & \multicolumn{2}{|c|}{ Difference in Vital Sign domain scores in the two conditions } \\
\hline Outcome measure (standard score) & Standing & Seated \\
\hline CNSVS domains & & $106 \pm 11$ \\
\hline Executive Function & $101 \pm 10$ & $99 \pm 9$ \\
\hline Complex Attention & $94 \pm 10$ & $104 \pm 12$ \\
\hline Cognitive Flexibility & $99 \pm 12$ & 0.18 \\
\hline Psychomotor Speed & $112 \pm 12$ & $0.01^{*}$ \\
\hline Reaction Time & $100 \pm 8$ & 0.17 \\
\hline Processing Speed & $111 \pm 10$ & $104 \pm 10$ \\
\hline
\end{tabular}

Table 1: The outcome scores of each of the six cognitive executive function domains tested in the two conditions and the between-condition p-value. Higher domain scores indicate better cognitive executive function performance. Results are means (SD). * indicate a significance level of $<0.05$.

optimization of the knowledge work environment a priority. For knowledge workers in offices or laboratories who must switch between selective attention for one task and a sustained focus for another, our results suggest there may be benefits in moving between seated and standing positions for specific task types. We may therefore ask, are there ways that we can help people identify the moments to shift selfposition (going from seated work to mobile cogitation and back) sooner and thus accelerate pace of breakthroughs? Understanding how task type may map to position to inform self-positioning choices could potentially help reduce effects of stress in cognitively taxing environments from nuclear control rooms [23] to medical ward performance. These findings open interesting possibilities for the design of information intense environments that combine new display technologies and sensors. For instance, interrupted work has been shown to have stressful consequences in knowledge-working environments, leading to higher levels of frustration and a requirement to work faster [24]. Near-future designs may support a knowledge worker's choice to have a standing desk gently begin to descend to a seated level on detection of multitasking vs. single task work. In the working environment, its ergonomic and cognitive aids are designed to support best possible cognitive executive function, we may find that long term stress and frustration is reduced, thus promoting mental health and improving performance in the working environment.

Our study has some limitations. One limitation was the customization of the CNSVS test battery leaving out the Visual and Verbal Memory Tests. Gualtieri and Johnson [25] showed high testrest reliability of the complete CNSVS test battery as well as within the individual domains. Because in our experimental design we ran the two test conditions on the same day for each participant the Visual and Verbal Memory Tests were excluded due to the shortterm learning effect these might produce. For the same reason, as well as a time efficiency standpoint, we also excluded Non-Verbal Reasoning and Social Acuity. Besides avoiding having a learning effect to take place, the underlying argumentation for excluding nonverbal social behaviour is found in the outcome measures of interest. The aforementioned subtests rely more on emotional perception than cognitive performance. While that is of great interest too, we decided to exclude those from this particular trial. In addition, not controlling for time of day is a limitation to this study because this may introduce some variation between individuals [26]. On the other hand, time of day may have minimal influence of differences between conditions as we used a paired design with randomization of order.

In terms of future work, our results on self-positioning effects on task performance raise further questions for optimizing cognitive performance. Breakthroughs to problems are often experienced when going for a walk or shampooing our heads in the shower, getting away from the seated, working memory focus of concentrating at the desk, in particular, it seems, not staying in a static position, focused on a static target like a screen. Indeed there is an entire literature devoted to understanding what is actually, formally called "the aha moment" in discovery and where/when these occur [27]. It may be that our knowledge work design needs to begin to find ways to accommodate and support work-in-motion.

In conclusion our randomized controlled cross-over trial on selfpositioning and cognitive performance showed significant higher scores in a domain of cognitive executive function known as Complex Attention but not in the other domains. Based on these initial results, we have shown that there is scope for further work in this area. For instance, information design in the workplace environment can be enhanced according to one's current cognitive requirements and thereby may optimize opportunities to produce better results, decrease the chance of making mistakes and lower the relative cognitive load of people working with tasks requiring a sustained focus and resistance to distraction

\section{References}

1. Hamilton MT, Hamilton DG, Zderic TW (2007) Role of Low Energy Expenditure and Sitting in Obesity, Metabolic Syndrome, Type 2 Diabetes, and Cardiovascular Disease. Diabetes 56: 2655-2667.

2. Katzmarzyk PT, Church TS, Craig CL, Bouchard C (2009) Sitting Time and Mortality from All Causes, Cardiovascular Disease, and Cancer. Med Sci Sports Exerc 41: 998-1005.

3. Callaghan JP, McGill SM (2001) Low Back Joint Loading and Kinematics During Standing and Unsupported Sitting. Ergonomics 44: 280-294.

4. O'Sullivan PB, Grahamslaw KB, Kendell M, Lapenskie SC, Möller NE, et al. (2002) The Effect of Different Standing and Sitting Postures on Trunk Muscle Activity in a Pain-free Population. Spine (Phila Pa 1976) 27: 1238-1244.

5. Bendix T, Krohn L, Jessen F, Aaras A (1985) Trunk posture and trapezius muscle load while working in standing, supported-standing, and sitting positions. Spine (Phila Pa 1976) 10: 433-439.

6. Nachemson A, Elfström G (1970) Intravital Dynamic Pressure Measurements in Lumbar Discs. A Study of Common Movements, Maneuvers and Exercises. Scand J Rehabil Med Suppl 1: 1-40.

7. Nachemson A (1975) Towards a Better Understanding of Low-back Pain: Review of the Mechanics of the Lumbar Disc. Rheumatol Rehabil 14: 129-143.

8. Andersson BJ, Ortengren R (1974) Lumbar Disc Pressure and Myoelectric Back Muscle Activity During Sitting. II. Studies on an Office Chair. Scand J of Rehabil Med 6: 115-121.

9. Occhipinti E, Colombini D, Frigo C, Pedotti A, Grieco A (1985) Sitting Posture: Analysis of Lumbar Stresses with Upper Limbs Supported. Ergonomics 28 1333-1346.

10. Husemann B, von Mach CY, Borsotto D, Zepf KI, Scharnbacher J (2009) Comparisons of Musculoskeletal Complaints and Data Entry Between a Sitting and a Sit-Stand Workstation Paradigm. Hum Factors 51: 310-320.

11. Brewer S, van Eerd D, Amick BC 3rd, Irvin E, Daum KM, et al. (2006) Workplace Interventions to Prevent Musculoskeletal and Visual Symptoms and Disorders Among Computer Users: a Systematic Review. J Occup Rehabil 16: 325-358.

12. Ebara T, Kubo T, Inoue T, Murasaki GI, Takeyama H, et al. (2008) Effects of 
Citation: Schraefel MC, Kenneth Jay, Andersen LL (2012) Assessing the Effect of Self-positioning on Cognitive Executive Function. J Ergonomics 2:110. doi:10.4172/2165-7556.1000110

Page 4 of 4

adjustable sit-stand vdt workstations on workers' musculoskeletal discomfort, alertness and performance. Ind Health 46: 497-505.

13. Miller EK, Freedman DJ, Wallis JD (2002) The Prefrontal Cortex: Categories, Concepts and Cognition. Philos Trans R Soc Lond B Biol Sci 357: 1123-1136.

14. Chan RC, Shum D, Toulopoulou T, Chen EY (2008) Assessment of Executive Functions: Review of Instruments and Identification of Critical Issues. Arch Clin Neuropsychol 23: 201-216.

15. Levine JA, Miller JM (2007) The energy expenditure of using a "walk-and-work" desk for office workers with obesity. Br J Sports Med 41: 558-561.

16. Ohlinger CM, Horn TS, Berg WP, Cox RH (2011) The Effect of Active Workstation Use on Measures of Cognition, Attention, and Motor Skill. J Phys Act Health 8: 119-125.

17. John D, Bassett D, Thompson D, Fairbrother J, Baldwin D (2009) Effect of Using a Treadmill Workstation on Performance of Simulated Office Work Tasks. J Phys Act Health 6: 617-624.

18. Reinhardt W, Schmidt B, Sloep P, Drachsler H (2011) Knowledge Worker Roles and Actions-Results of Two Empirical Studies. Knowledge and Process Management 18: 150-174.

19. Plebani M, Carraro $P$ (1997) Mistakes in a Stat Laboratory: Types and Frequency. Clin Chem 43: 1348-1351.
20. Jequier AM, Ukombe EB (1983) Errors Inherent in the Performance of a Routine Semen Analysis. British Journal of Urology 55: 434-436.

21. Kok, Wim (2004) Facing the Challenge - The Lisbon Strategy for Growth and Employment. Report from the High Level Group.

22. Brinkley I (2008) The Knowledge Economy: How Knowledge is Reshaping the Economic Life of Nations.

23. Vicente KJ (2004) The human factor : revolutionizing the way people live with technology. (1stedn), New York: Routledge.

24. Gloria M, Gudith D, Klocke U (2008) The Cost of Interrupted Work: more speed and stress. In: Proceeding of the Twenty-sixth Annual $\mathrm{CHI}$ Conference on Human Factors in Computing Systems-CHI '08, 107. New York, USA.

25. Gualtieri CT, Johnson LG (2006) Reliability and Validity of a Computerized Neurocognitive Test Battery, CNS Vital Signs. Arch Clin Neuropsychol 21: 623643.

26. Ruddle RA, Volkova E, Bülthoff BH (2011) Walking improves your cognitive map in environments that are large-scale and large in extent. ACM Transactions on Computer-Human Interaction 18: 1-20.

27. Kounios J, Frymiare JL, Bowden EM, Fleck JI, Subramaniam K, et al. (2006) The Prepared Mind: Neural Activity Prior to Problem Presentation Predicts Subsequent Solution by Sudden Insight. Psychol Sci 17: 882-890.
Submit your next manuscript and get advantages of OMICS Group submissions

Unique features:

- User friendly/feasible website-translation of your paper to 50 world's leading languages

- Audio Version of published paper

Digital articles to share and explore

Special features:

- 200 Open Access Journals

15,000 editorial team

21 days rapid review process

Quality and quick editorial, review and publication processing

Indexing at PubMed (partial), Scopus, DOAJ, EBSCO, Index Copernicus and Google Scholar etc

Sharing Option: Social Networking Enabled

- Authors, Reviewers and Editors rewarded with online Scientific Credits

- Better discount for your subsequent articles

Submit your manuscript at: www.omicsonline.org/submission 